PROCEEDINGS OF THE

AMERICAN MATHEMATICAL SOCIETY

Volume 140, Number 6, June 2012, Pages 2091-2102

S 0002-9939(2011)11076-1

Article electronically published on October 20, 2011

\title{
A NOTE ON THE CONE RESTRICTION CONJECTURE
}

\author{
CHANGXING MIAO, JUNYONG ZHANG, AND JIQIANG ZHENG
}

(Communicated by Hart F. Smith)

\begin{abstract}
This paper is devoted to the study of the restriction problem in harmonic analysis. Based on the spherical harmonics expansion and analyzing the asymptotic behavior of the Bessel function, we show that a modified linear adjoint restriction estimate holds for all Schwartz functions compactly supported on the cone, which generalizes Shao's result.
\end{abstract}

\section{INTRODUCTION AND STATEMENT OF MAIN RESULT}

Stein [5] first considered the restriction problem for the sphere case, and then this problem was naturally extended to smooth submanifolds of $\mathbb{R} \times \mathbb{R}^{n}$ with appropriate curvature [6] such as the paraboloid and the cone. For recent progress on the restriction problem, we refer readers to the survey [10].

In this paper, we shall focus our attention on the set $S$ which is a nonempty smooth compact subset of the cone,

$$
\left\{(\tau, \xi) \in \mathbb{R} \times \mathbb{R}^{n}: \tau=|\xi|\right\} \quad \text { with } \quad n \geq 2 .
$$

In order to restrict the Fourier transform of an $L_{t, x}^{q^{\prime}}\left(\mathbb{R} \times \mathbb{R}^{n}\right)$-function to $S$ by a limiting argument, it suffices to prove an a priori "restriction estimate" of the form

$$
\|\hat{g}\|_{L^{p^{\prime}}(S ; \mathrm{d} \sigma)} \leq C_{p, q, n, S}\|g\|_{L_{t, x}^{q^{\prime}}\left(\mathbb{R} \times \mathbb{R}^{n}\right)}
$$

for all Schwartz functions $g$ and some $1 \leq p^{\prime} \leq \infty$, where the Fourier transform is defined by

$$
\hat{g}(\tau, \xi)=\int_{\mathbb{R} \times \mathbb{R}^{n}} e^{-2 \pi i(t \tau+x \cdot \xi)} g(t, x) \mathrm{d} t \mathrm{~d} x
$$

and the canonical measure $\mathrm{d} \sigma$ is the pull-back of the measure $\frac{\mathrm{d} \xi}{|\xi|}$ under the projection map $(\tau, \xi) \mapsto \xi$. By duality, the estimate (1.1) is equivalent to the following extension estimate for the cone:

$$
\left\|(f \mathrm{~d} \sigma)^{\vee}\right\|_{L_{t, x}^{q}\left(\mathbb{R} \times \mathbb{R}^{n}\right)} \leq C_{p, q, n, S}\|f\|_{L^{p}(S, \mathrm{~d} \sigma)}
$$

Received by the editors September 15, 2010 and, in revised form, January 18, 2011 and February 11,2011

2010 Mathematics Subject Classification. Primary 35Q40, 35Q55, 47J35.

Key words and phrases. Linear adjoint restriction estimate, spherical harmonics. 
for all Schwartz functions $f$, where

$$
\begin{aligned}
(f \mathrm{~d} \sigma)^{\vee}(t, x) & =\int_{S} f(\tau, \xi) e^{2 \pi i(x \cdot \xi+t \tau)} \mathrm{d} \sigma(\xi) \\
& =\int_{\mathbb{R}^{n}} f(|\xi|, \xi) e^{2 \pi i(x \cdot \xi+t|\xi|)} \frac{\mathrm{d} \xi}{|\xi|}
\end{aligned}
$$

denotes the inverse space-time Fourier transform of the measure $f \mathrm{~d} \sigma$. The restriction conjecture asks for the optimal choice of the range of exponents $p$ and $q$ for which the estimate (1.2) holds.

By testing (1.2) with the characteristic functions supported on a symmetric band, we have realized that $q>\frac{2 n}{n-1}$ is one necessary condition for (1.2). On the other hand, the Knapp counterexample shows that we need another necessary requirement $\frac{n+1}{q} \leq \frac{n-1}{p^{\prime}}$. Hence, the conjecture on the restriction of the Fourier transform to the cone can be stated as follows.

Conjecture 1.1. The inequality (1.2) holds with constants depending on $n, p, q$ and $S$ if and only if $q>\frac{2 n}{n-1}$ and $\frac{n+1}{q} \leq \frac{n-1}{p^{\prime}}$.

There is a large amount of literature focused on this problem. When $p=2$, Córdoba and Stein have proved that (1.2) was true if $q \geq \frac{2(n+1)}{n-1}$ in an unpublished work. Strichartz 8 then has extended the result to more general quadratic surfaces. Barcelo [1] has proved Conjecture 1.1 when $n=2$. Based on a new bilinear cone restriction estimate, Wolff [16] recently showed a milestone result: the conjecture was true when $n=3$ and gave the best known result $q>\frac{2(n+3)}{n+1}$ in higher dimensions $n \geq 4$. To prove the best restriction estimates known to date, some fantastic tools such as the so-called bilinear method, the wave packet decomposition and the induction-on-scales were explored and developed. For details, one can refer to 10, 12, 13, 14, 16.

Very recently, Shao 4 provided two simple and novel arguments to prove that Conjecture 1.1 holds for the cylindrically symmetric functions which are supported on the cone $S$. We observe that, if the test function is radial (namely, invariant under the spatial rotation), then all of the $L^{q}$ norms in the angular variable of the test function are equivalent without considering some harmless constant. Motivated by this observation, it is tempting to remove the cylindrically symmetric assumption by substituting the $L_{t, x}^{q}$ norm in (1.2) with the $L_{t}^{q}\left(\mathbb{R} ; L_{r^{n-1} \mathrm{~d} r}^{q} L_{\theta}^{\tilde{q}}\left(\mathbb{S}^{n-1}\right)\right)$ norm with $\tilde{q} \leq q$. Of course, if one could prove (1.4) (see below) with $\tilde{q}=q$, then one could obtain the conjecture. In this paper, we utilize the spherical harmonics expansion, the property of the Bessel function and a variant of the argument in Nicola 2 and Shao 4 to prove the following theorem:

Theorem 1.1. Suppose $q>\frac{2 n}{n-1}, 1 \leq \tilde{q} \leq 2$ and $\frac{n+1}{q} \leq \frac{n-1}{p^{\prime}}$. Then

$$
\left\|(f \mathrm{~d} \sigma)^{\vee}\right\|_{L_{t}^{q}\left(\mathbb{R} ; L_{r n-1}^{q} \mathrm{~d} r\right.} L_{\theta}^{\left.\tilde{q}\left(\mathbb{S}^{n-1}\right)\right)} \leq C_{p, q, n, S}\|f\|_{L^{p}(S, \mathrm{~d} \sigma)}
$$

holds for all Schwartz functions $f$.

Remark 1.1. Since $q>\frac{2 n}{n-1}>2$, the Hölder inequality implies that the estimate (1.4) in Theorem 1.1 is weaker than the Conjecture 1.1 
Remark 1.2. The estimate (1.4) generalizes Shao's result in [4. Actually, if $f$ is a cylindrically symmetric function, so is $(f \mathrm{~d} \sigma)^{\vee}(t, x)$. Then

$$
\begin{aligned}
\left\|(f \mathrm{~d} \sigma)^{\vee}\right\|_{L_{t, x}^{q}\left(\mathbb{R} \times \mathbb{R}^{n}\right)} & =\left\|(f \mathrm{~d} \sigma)^{\vee}\right\|_{L_{t}^{q}\left(\mathbb{R} ; L_{r^{n-1} \mathrm{~d} r}^{q} L_{\theta}^{q}\left(\mathbb{S}^{n-1}\right)\right)} \\
& \simeq\left\|(f \mathrm{~d} \sigma)^{\vee}\right\|_{L_{t}^{q}\left(\mathbb{R} ; L_{r^{n-1} \mathrm{~d} r}^{q} L_{\theta}^{2}\left(\mathbb{S}^{n-1}\right)\right) .}
\end{aligned}
$$

Now we introduce some notation. We use $A \lesssim B$ to denote the statement that $A \leq C B$ for some large constant $C$ which may vary from line to line and depend on various parameters, and similarly use $A \ll B$ to denote the statement $A \leq C^{-1} B$. We employ $A \sim B$ to denote the statement that $A \lesssim B \lesssim A$. If the constant $C$ depends on a special parameter other than the above, we shall denote it explicitly by subscripts. For instance, $C_{\epsilon}$ should be understood as a positive constant not only depending on $p, q, n$ and $S$, but also on $\epsilon$. Throughout this paper, pairs of conjugate indices are written as $p, p^{\prime}$, where $\frac{1}{p}+\frac{1}{p^{\prime}}=1$ with $1 \leq p \leq \infty$.

This paper is organized as follows: In section 2 , we present some simple facts about the Hankel transforms, and the Bessel functions and recall the Van der Corput lemma that will be useful in the sequel section. Section 3 is devoted to the proof of Theorem 1.1] via the spherical harmonics expansion and analyzing the asymptotic behavior of the Bessel function.

\section{Preliminaries}

In this section, we list some simple and standard facts about the Hankel transforms and the Bessel functions. At the end of this section, we recall the Van der Corput lemma that will be useful in the next section.

We begin with recalling the expansion formula with respect to the spherical harmonics. For more details, we refer to Stein-Weiss [7. For the sake of convenience, we let

$$
\xi=\rho \omega \quad \text { and } \quad x=r \theta \quad \text { with } \quad \omega, \theta \in \mathbb{S}^{n-1} .
$$

For any $g \in L^{2}\left(\mathbb{R}^{n}\right)$, the expansion formula with respect to the spherical harmonics yields

$$
g(\xi)=\sum_{k=0}^{\infty} \sum_{\ell=1}^{d(k)} a_{k, \ell}(\rho) Y_{k, \ell}(\omega),
$$

where

$$
\left\{Y_{k, 1}, \ldots, Y_{k, d(k)}\right\}
$$

is the orthogonal basis of the space of spherical harmonics of degree $k$ on $\mathbb{S}^{n-1}$, called $\mathcal{H}^{k}$, with the dimension

$$
d(k)=\frac{2 k+n-2}{k} C_{n+k-3}^{k-1} \simeq\langle k\rangle^{n-2} .
$$

We remark that in the case of $n=2$, the dimension of $\mathcal{H}^{k}$ is a constant, which is independent of $k$. Obviously, we have the orthogonal decomposition of

$$
L^{2}\left(\mathbb{S}^{n-1}\right)=\bigoplus_{k=0}^{\infty} \mathcal{H}^{k}
$$

This gives that by orthogonality

$$
\|g(\xi)\|_{L_{\omega}^{2}}=\left\|a_{k, \ell}(\rho)\right\|_{\ell_{k, \ell}^{2}} \cdot
$$


For our purpose, we need to recall the inverse Fourier transform of $a_{k, \ell}(\rho) Y_{k, \ell}(\omega)$. Theorem 3.10 in [7] asserts the Hankel transforms formula that

$$
\check{g}(r \theta)=\sum_{k=0}^{\infty} \sum_{\ell=1}^{d(k)} 2 \pi i^{k} Y_{k, \ell}(\theta) r^{-\frac{n-2}{2}} \int_{0}^{\infty} J_{k+\frac{n-2}{2}}(2 \pi r \rho) a_{k, \ell}(\rho) \rho^{\frac{n}{2}} \mathrm{~d} \rho,
$$

where the Bessel function $J_{k}(r)$ of order $k$ is defined by the integral

$$
J_{k}(r)=\frac{(r / 2)^{k}}{\Gamma\left(k+\frac{1}{2}\right) \Gamma(1 / 2)} \int_{-1}^{1} e^{i s r}\left(1-s^{2}\right)^{(2 k-1) / 2} \mathrm{~d} s \quad \text { with } k>-\frac{1}{2} \text { and } r>0 .
$$

A simple computation gives the rough estimate

$$
\left|J_{k}(r)\right| \leq \frac{C r^{k}}{2^{k} \Gamma\left(k+\frac{1}{2}\right) \Gamma(1 / 2)}\left(1+\frac{1}{k+1 / 2}\right),
$$

with the absolute constant $C$ and this estimate will be mainly used when $r \lesssim 1$. Another well-known asymptotic expansion about the Bessel function is

$$
J_{k}(r)=r^{-1 / 2} \sqrt{\frac{2}{\pi}} \cos \left(r-\frac{k \pi}{2}-\frac{\pi}{4}\right)+O_{k}\left(r^{-3 / 2}\right), \quad \text { as } r \rightarrow \infty
$$

but with a constant which would depend on $k$ (see [7]). As pointed out in [6], if one seeks a uniform bound for large $r$ and $k$, then the best one can do is $\left|J_{k}(r)\right| \leq C r^{-\frac{1}{3}}$. To effectively utilize the oscillatory behavior of $e^{2 \pi i t \rho}$ to investigate the behavior of the asymptotic bound on $k$ and $r$ in the next section, we would like to recall Schläfli's integral representation [15] of the Bessel function: for $r \in \mathbb{R}^{+}$and $k>-\frac{1}{2}$,

$$
\begin{aligned}
J_{k}(r) & =\frac{1}{2 \pi} \int_{-\pi}^{\pi} e^{i r \sin \theta-i k \theta} \mathrm{d} \theta-\frac{\sin (k \pi)}{\pi} \int_{0}^{\infty} e^{-(r \sinh s+k s)} \mathrm{d} s \\
& :=\tilde{J}_{k}(r)-E_{k}(r) .
\end{aligned}
$$

We will be interested only in the case $k \in \frac{1}{2} \mathbb{N}$. We remark that $E_{k}(r)=0$ when $k \in \mathbb{Z}^{+}$. By a computation of the fundamental integral, this gives that for $r>0$,

$$
\left|E_{k}(r)\right|=\left|\frac{\sin (k \pi)}{\pi} \int_{0}^{\infty} e^{-(r \sinh s+k s)} \mathrm{d} s\right| \leq C(r+k)^{-1} .
$$

We conclude this section by recalling Van der Corput's lemma [6].

Lemma 2.1. Let $\phi$ be a smooth real-valued function defined on an interval $[a, b]$ and let $\left|\phi^{(k)}(x)\right| \geq 1$ for all $x \in[a, b]$. Then

$$
\left|\int_{a}^{b} e^{i \lambda \phi(x)} \mathrm{d} x\right| \leq c_{k} \lambda^{-\frac{1}{k}}
$$

holds when:

- $k \geq 2$ or

- $k=1$ and $\phi^{\prime}(x)$ is monotonic.

The bound $c_{k}$ is independent of $\phi$ and $\lambda$. 


\section{Proof of Theorem 1.1}

In this section, we will provide the proof of Theorem 1.1. The argument is organized as follows. We first utilize the scaling argument to reduce ourselves to considering only the unit cap supported test function $f$. Secondly we use the Hankel transforms formula to write $(f \mathrm{~d} \sigma)^{\vee}$ as an expression in terms of spherical harmonics. Due to the behavior of the Bessel function, we separately investigate the behavior of $(f \mathrm{~d} \sigma)^{\vee}$ on $\{x:|x| \leq 1\}$ and $\{x:|x| \geq 1\}$. Then we focus on the main term involving $\tilde{J}_{k}(r)$ on the latter region. To handle this complicated case, we divide $\tilde{J}_{k}(r)$ into three pieces due to the Van der Corput lemma. We handle the first two subcases by making use of the Van der Corput lemma and the HausdorffYoung inequality. To deal with the third subcase, since the Bessel function decays at a lower rate, we need more oscillatory behavior from $e^{2 \pi i t \rho}$ to overcome this difficulty. To this end, in the spirit of J. Sterbenz [9] we investigate the asymptotic behavior of the function $\psi_{t-\frac{j}{4}}^{k}(r)$ (defined in (3.18) below).

We now turn to the details. The proof of Theorem 1.1 will focus on the case where $p \geq 2$, as the $p \leq 2$ case follows from the Tomas-Stein inequality [6], or the result in Strichartz [8]. Precisely, we will establish

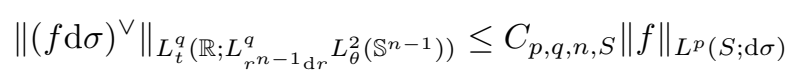

for all Schwartz functions $f$, under the conditions $q>\frac{2 n}{n-1}$ and $\frac{n+1}{q}=\frac{n-1}{p^{\prime}}$ with $p \geq 2$. We now reduce ourselves to proving (3.1) for the unit cap supported $f$ via the scaling argument. For the general Schwartz function $f$, we decompose it as follows:

$$
f=\sum_{M} f_{M}
$$

where $M \in 2^{\mathbb{Z}}$ and $f_{M}=f 1_{\{(\tau, \xi): \tau=|\xi|, M \leq|\xi| \leq 2 M\}}$. By the scaling argument, we have

$$
\begin{aligned}
& \left.\left\|(f \mathrm{~d} \sigma)^{\vee}\right\|_{L_{t}^{q}\left(\mathbb{R} ; L_{r n-1}^{q} \mathrm{~d} r\right.} L_{\theta}^{2}\left(\mathbb{S}^{n-1}\right)\right) \\
& \left.\leq\left(\sum_{R}\left(\sum_{M}\left\|\left(f_{M} \mathrm{~d} \sigma\right)^{\vee}(t, x)\right\|_{L_{t}^{q}\left(\mathbb{R} ; L_{r^{n-1} \mathrm{~d} r}^{q}\right.}([R, 2 R]) L_{\theta}^{2}\left(\mathbb{S}^{n-1}\right)\right)\right)^{q}\right)^{1 / q} \\
& \left.\leq\left(\sum_{R}\left(\sum_{M} M^{(n-1)-\frac{n+1}{q}}\left\|\left(f_{1} \mathrm{~d} \sigma\right)^{\vee}(t, x)\right\|_{L_{t}^{q}\left(\mathbb{R} ; L_{r^{n-1} \mathrm{~d} r}^{q}\right.}([M R, 2 M R]) L_{\theta}^{2}\left(\mathbb{S}^{n-1}\right)\right)\right)^{q}\right)^{1 / q},
\end{aligned}
$$

where $R \in 2^{\mathbb{Z}}$. Thus if we could show for $q \geq 2$ and $R \in 2^{\mathbb{Z}}$ that

$$
\begin{aligned}
& \left.\left\|(f \mathrm{~d} \sigma)^{\vee}\right\|_{L_{t}^{q}\left(\mathbb{R} ; L_{r^{n-1} \mathrm{~d} r}^{q}\right.}([R, 2 R]) L_{\theta}^{2}\left(\mathbb{S}^{n-1}\right)\right) \\
& \quad \leq C_{q, n, S} \min \left\{R^{\frac{n}{q}}, R^{-\frac{n-1}{2}\left(1-\frac{2 n}{q(n-1)}\right)}\right\}\|f\|_{L^{2}(S ; \mathrm{d} \sigma)}
\end{aligned}
$$

for all Schwartz functions $f$ supported on a cap of the form

$$
\{(\tau, \xi): \tau=|\xi|, 1 \leq|\xi| \leq 2\},
$$


then we could utilize $\frac{n+1}{q}=\frac{n-1}{p^{\prime}}$ to obtain

$$
\begin{aligned}
& \left.\left\|(f \mathrm{~d} \sigma)^{\vee}\right\|_{L_{t}^{q}\left(\mathbb{R} ; L_{r n-1}^{q} \mathrm{~d} r\right.} L_{\theta}^{2}\left(\mathbb{S}^{n-1}\right)\right) \\
& \leq\left(\sum_{R}\left(\sum_{M} \min \left\{(R M)^{\frac{n}{q}},(R M)^{-\frac{n-1}{2}\left(1-\frac{2 n}{q(n-1)}\right)}\right\} M^{(n-1)-\frac{n+1}{q}}\left\|f_{1}\right\|_{L^{p}(S ; \mathrm{d} \sigma)}\right)^{q}\right)^{1 / q} \\
& \leq\left(\sum_{R}\left(\sum_{M} \min \left\{(R M)^{\frac{n}{q}},(R M)^{-\frac{n-1}{2}\left(1-\frac{2 n}{q(n-1)}\right)}\right\}\left\|f_{M}\right\|_{L^{p}(S ; \mathrm{d} \sigma)}\right)^{q}\right)^{1 / q} .
\end{aligned}
$$

By $q>\frac{2 n}{n-1}$, it follows that

$$
\sup _{R} \sum_{M} \min \left\{(R M)^{\frac{n}{q}},(R M)^{-\frac{n-1}{2}\left(1-\frac{2 n}{q(n-1)}\right)}\right\}<\infty
$$

and

$$
\sup _{M} \sum_{R} \min \left\{(R M)^{\frac{n}{q}},(R M)^{-\frac{n-1}{2}\left(1-\frac{2 n}{q(n-1)}\right)}\right\}<\infty .
$$

By making use of Schur's test lemma and $\ell^{p} \hookrightarrow \ell^{q}$ with $q>\frac{2 n}{n-1}>p$, we have

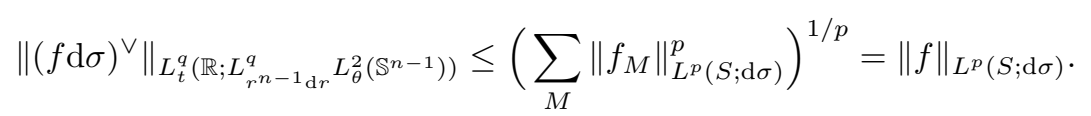

Hence it remains to prove (3.2). This will be done in the rest of this paper. By interpolation, it suffices to prove

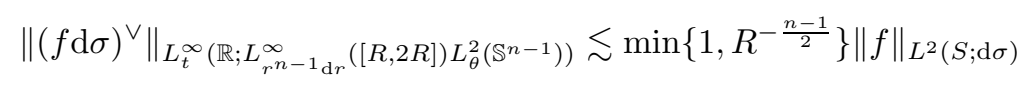

and

$$
\left.\left\|(f \mathrm{~d} \sigma)^{\vee}\right\|_{L_{t}^{2}\left(\mathbb{R} ; L_{r n-1}^{2} \mathrm{~d} r\right.}([R, 2 R]) L_{\theta}^{2}\left(\mathbb{S}^{n-1}\right)\right) \lesssim \min \left\{R^{\frac{n}{2}}, R^{\frac{1}{2}}\right\}\|f\|_{L^{2}(S ; \mathrm{d} \sigma)} .
$$

To this end, we rewrite

$$
(f(\tau, \xi) \mathrm{d} \sigma)^{\vee}(t, x)=\int_{\mathbb{R}^{n}} g(\xi) e^{2 \pi i(x \cdot \xi+t|\xi|)} \mathrm{d} \xi,
$$

where

$$
g(\xi)=f(|\xi|, \xi)|\xi|^{-1} \in \mathcal{S}\left(\mathbb{R}^{n}\right)
$$

with supp $g \subset\{\xi:|\xi| \in[1,2]\}$. Putting the spherical harmonics expansion

$$
g(\xi)=\sum_{k=0}^{\infty} \sum_{\ell=1}^{d(k)} a_{k, \ell}(\rho) Y_{k, \ell}(\omega)
$$

into (3.5) and using the Hankel transforms formula (2.3), we expand the expression (3.5) in terms of spherical harmonics as

$(f(\tau, \xi) \mathrm{d} \sigma)^{\vee}(t, x)=\sum_{k=0}^{\infty} \sum_{\ell=1}^{d(k)} 2 \pi i^{k} r^{-\frac{n-2}{2}} \int_{0}^{\infty} e^{-2 \pi i t \rho} J_{k+\frac{n-2}{2}}(2 \pi r \rho) a_{k, \ell}(\rho) \rho^{\frac{n}{2}} \mathrm{~d} \rho Y_{k, \ell}(\theta)$.

Noting that $a_{k, \ell}(\rho)$ is supported on $[1,2]$ and setting $k_{n}:=k+\frac{n-2}{2}$, we can further write

$$
(f \mathrm{~d} \sigma)^{\vee}(t, x)=\sum_{k=0}^{\infty} \sum_{\ell=1}^{d(k)} 2 \pi i^{k} r^{-\frac{n-2}{2}} \int_{0}^{\infty} e^{-2 \pi i t \rho} J_{k_{n}}(2 \pi r \rho) a_{k, \ell}(\rho) \varphi(\rho) \mathrm{d} \rho Y_{k, \ell}(\theta),
$$


where $\varphi$ is a smooth bump function supported on the interval $\left(\frac{1}{2}, 4\right)$ and $\rho^{\frac{n}{2}}$ is absorbed by the function $\varphi$. To use effectively the oscillation of $e^{2 \pi i t \rho}$, in the spirit of J. Sterbenz [9] we write the Fourier series of $a_{k, \ell}(\rho)$ as

$$
a_{k, \ell}(\rho)=\sum_{j} b_{k, \ell}^{j} e^{i \frac{\pi}{2} \rho j} \quad \text { with } \quad b_{k, \ell}^{j}=\frac{1}{4} \int_{0}^{4} e^{-i \frac{\pi}{2} \rho j} a_{k, \ell}(\rho) \mathrm{d} \rho .
$$

Thus it follows that

$$
\begin{aligned}
& (f \mathrm{~d} \sigma)^{\vee}(t, x) \\
& \quad=\sum_{k=0}^{\infty} \sum_{\ell=1}^{d(k)} 2 \pi i^{k} r^{-\frac{n-2}{2}} \int_{0}^{\infty} e^{-2 \pi i t \rho} J_{k_{n}}(2 \pi r \rho) \sum_{j} b_{k, \ell}^{j} e^{i \frac{\pi}{2} \rho j} \varphi(\rho) \mathrm{d} \rho Y_{k, \ell}(\theta) .
\end{aligned}
$$

By the Plancherel theorem and the orthogonality, we obtain

$$
\left\|(f \mathrm{~d} \sigma)^{\vee}(t, x)\right\|_{L_{x}^{2}}=\|g\|_{L_{\xi}^{2}} \cong\left(\sum_{k=0}^{\infty} \sum_{\ell=1}^{d(k)} \sum_{j}\left|b_{k, \ell}^{j}\right|^{2}\right)^{\frac{1}{2}} .
$$

Since $\|g\|_{L_{\xi}^{2}} \sim\|f\|_{L^{2}(S ; \mathrm{d} \sigma)}$, we can normalize $\|g\|_{L_{\xi}^{2}}=1$. By the orthogonality of $\left\{Y_{k, \ell}(\theta)\right\}$, we can estimate $(f \mathrm{~d} \sigma)^{\vee}(t, x)$ on the region $\{x:|x| \leq 1\}$ via the following proposition.

Proposition 3.1. We have the following estimate for $q \geq 2, R \leq 1$ :

$$
\begin{aligned}
&\left.\left\|r^{-\frac{n-2}{2}}\left(\sum_{k=0}^{\infty} \sum_{\ell=1}^{d(k)}\left|\int_{0}^{\infty} e^{-2 \pi i t \rho} J_{k_{n}}(2 \pi r \rho) \sum_{j} b_{k, \ell}^{j} e^{i \frac{\pi}{2} \rho j} \varphi(\rho) \mathrm{d} \rho\right|^{2}\right)^{\frac{1}{2}}\right\|_{L_{t}^{q}\left(\mathbb{R} ; L_{r^{n-1} \mathrm{~d} r}^{q}\right.}([R, 2 R])\right) \\
& \lesssim R^{\frac{n}{q}}
\end{aligned}
$$

Proof. Since $q \geq 2$, by making use of the Minkowski inequality and the HausdorffYoung inequality in $t$, we obtain that

L.H.S. of (3.8)

$$
\lesssim\left\|r^{-\frac{n-2}{2}}\left(\sum_{k=0}^{\infty} \sum_{\ell=1}^{d(k)}\left\|J_{k_{n}}(2 \pi r \rho) \sum_{j} b_{k, \ell}^{j} e^{i \frac{\pi}{2} \rho j} \varphi(\rho)\right\|_{L_{\rho}^{q^{\prime}}}^{2}\right)^{\frac{1}{2}}\right\|_{L_{r^{n-1} \mathrm{~d} r}^{q}([R, 2 R])} .
$$

Since supp $\varphi \subset\left[\frac{1}{2}, 4\right]$, it follows from Hölder's inequality and (2.4) that

L.H.S. of (3.8)

$$
\begin{aligned}
& \lesssim\left(\int_{R}^{2 R} r^{-\frac{(n-2) q}{2}}\left(\sum_{k=0}^{\infty} \sum_{\ell=1}^{d(k)}\left|\frac{(8 \pi r)^{k_{n}}}{2^{k_{n}} \Gamma\left(k_{n}+\frac{1}{2}\right) \Gamma(1 / 2)}\right|^{2}\left\|\sum_{j} b_{k, \ell}^{j} e^{i \frac{\pi}{2} \rho j}\right\|_{L_{\rho}^{2}}^{2}\right)^{\frac{q}{2}} r^{n-1} \mathrm{~d} r\right)^{\frac{1}{q}} \\
& \lesssim R^{\frac{n}{q}}
\end{aligned}
$$

where we make use of $k_{n}=k+\frac{n-2}{2}>0$ and $\Gamma(x+1)=x \Gamma(x)$. This proves Proposition 3.1 .

Then Proposition 3.1 gives (3.3) and (3.4) when $R \leq 1$. 
So we are left to prove (3.3) and (3.4) when $R \geq 1$. We first prove that (3.4) holds when $R \geq 1$. By (3.5), it suffices to prove

$$
\left\|\int_{\mathbb{R}^{n}} g(\xi) e^{2 \pi i(x \cdot \xi+t|\xi|)} \mathrm{d} \xi\right\|_{L_{t}^{2}\left(\mathbb{R} ; L_{x}^{2}(|x| \sim R)\right)} \lesssim R^{\frac{1}{2}}\|g\|_{L_{\xi}^{2}} .
$$

Recalling supp $g \subset\{\xi:|\xi| \in[1,2]\}$, we may assume $\left|\xi_{n}\right| \sim 1$. Then we freeze one spatial variable, say $x_{n}$, with $\left|x_{n}\right| \lesssim R$ and free other spatial variables $x^{\prime}=$ $\left(x_{1}, \ldots, x_{n}\right)$. After making the change of variables $\eta_{j}=\xi_{j}, \eta_{n}=|\xi|$ with $j=$ $1, \ldots, n-1$, we use the Plancherel theorem on the spacetime Fourier transform in $\left(t, x^{\prime}\right)$ to obtain (3.9).

To prove that (3.3) holds for $R \geq 1$, we need to establish the following proposition:

Proposition 3.2. We have the following estimates for $q \geq 2, R \geq 1$ :

$$
\begin{gathered}
\left\|r^{-\frac{n-2}{2}}\left(\sum_{k=0}^{\infty} \sum_{\ell=1}^{d(k)}\left|\int_{0}^{\infty} e^{-2 \pi i t \rho} J_{k_{n}}(2 \pi r \rho) \sum_{j} b_{k, \ell}^{j} e^{i \frac{\pi}{2} \rho j} \varphi(\rho) \mathrm{d} \rho\right|^{2}\right)^{\frac{1}{2}}\right\|_{L_{t}^{\infty}\left(\mathbb{R} ; L_{r^{n-1} \mathrm{~d} r}^{\infty}([R, 2 R])\right)} \\
\lesssim R^{-\frac{n-1}{2}} .
\end{gathered}
$$

Proof. To prove (3.10), we utilize Schläfli's integral representation of the Bessel function (2.5) to write $J_{k_{n}}(2 \pi r \rho)=E_{k_{n}}(2 \pi r \rho)+\tilde{J}_{k_{n}}(2 \pi r \rho)$. For simplicity, we set $q \geq 2$ from now on. Using (2.6), the Minkowski inequality and the Hausdorff-Young inequality in $t$ as before gives that

$$
\begin{gathered}
\left.\left\|r^{-\frac{n-2}{2}}\left(\sum_{k=0}^{\infty} \sum_{\ell=1}^{d(k)}\left|\int_{0}^{\infty} e^{-2 \pi i t \rho} E_{k_{n}}(2 \pi r \rho) \sum_{j} b_{k, \ell}^{j} e^{i \frac{\pi}{2} \rho j} \varphi(\rho) \mathrm{d} \rho\right|^{2}\right)^{\frac{1}{2}}\right\|_{L_{t}^{q}\left(\mathbb{R} ; L_{r n-1}^{q} \mathrm{~d} r\right.}([R, 2 R])\right) \\
\lesssim\left(\int_{R}^{2 R} r^{n(1-q / 2)-1} \mathrm{~d} r\right)^{\frac{1}{q}} \lesssim R^{-n\left(\frac{1}{2}-\frac{1}{q}\right)}
\end{gathered}
$$

Thus it remains to prove (3.10) replacing $J_{k_{n}}$ by $\tilde{J}_{k_{n}}$. In order to effectively use the Van der Corput Lemma 2.1 to investigate the behavior of $\tilde{J}_{k}(r)$, we decompose $[-\pi, \pi]$ into three partitions as follows:

$$
[-\pi, \pi]=I_{1} \cup I_{2} \cup I_{3},
$$

where

$$
I_{1}=\{\theta:|\theta| \leq \delta\}, \quad I_{2}=\left[-\pi,-\frac{\pi}{2}-\delta\right] \cup\left[\frac{\pi}{2}+\delta, \pi\right], \quad I_{3}=[-\pi, \pi] \backslash\left(I_{1} \cup I_{2}\right),
$$

with $0<\delta \ll 1$. We define

$$
\Phi_{r, k}(\theta)=\sin \theta-k \theta / r,
$$

and $\chi_{\delta}(\theta)$ is a smooth function given by

$$
\chi_{\delta}(\theta)= \begin{cases}1, & \theta \in[-\delta, \delta] ; \\ 0, & \theta \notin[-2 \delta, 2 \delta] .\end{cases}
$$


We then split $\tilde{J}_{k}(r)$ into three pieces and write

$$
\begin{aligned}
\tilde{J}_{k}(r) & =\frac{1}{2 \pi} \int_{-\pi}^{\pi} e^{i r \Phi_{r, k}(\theta)} \mathrm{d} \theta \\
& =\frac{1}{2 \pi}\left(\int_{-\pi}^{\pi} e^{i r \Phi_{r, k}(\theta)} \chi_{\delta}(\theta) \mathrm{d} \theta+\int_{I_{2}} e^{i r \Phi_{r, k}(\theta)} \mathrm{d} \theta+\int_{I_{3}} e^{i r \Phi_{r, k}(\theta)}\left(1-\chi_{\delta}(\theta)\right) \mathrm{d} \theta\right) \\
& :=\tilde{J}_{k}^{1}(r)+\tilde{J}_{k}^{2}(r)+\tilde{J}_{k}^{3}(r) .
\end{aligned}
$$

- Contribution of the case $\theta \in I_{2}$. In this case, the function $\Phi_{r, k}^{\prime}(\theta)=\cos \theta-k / r$ is monotonic in the intervals $\left[-\pi,-\frac{\pi}{2}-\delta\right]$ and $\left[\frac{\pi}{2}+\delta, \pi\right]$ respectively and satisfies that

$$
\left|\Phi_{r, k}^{\prime}(\theta)\right| \geq k / r+|\cos \theta| \geq \sin \delta .
$$

Then by Lemma 2.1, we have

$$
\left|\frac{1}{2 \pi} \int_{I_{2}} e^{i r \Phi_{r, k}(\theta)} \mathrm{d} \theta\right| \leq c_{\delta} r^{-1}
$$

uniformly in $k$. As before, the Minkowski inequality and the Hausdorff-Young inequality in $t$ show that

$$
\begin{aligned}
& \left.\left\|r^{-\frac{n-2}{2}}\left(\sum_{k=0}^{\infty} \sum_{\ell=1}^{d(k)}\left|\int_{0}^{\infty} e^{-2 \pi i t \rho} \tilde{J}_{k_{n}}^{2}(2 \pi r \rho) \sum_{j} b_{k, \ell}^{j} e^{i \frac{\pi}{2} \rho j} \varphi(\rho) \mathrm{d} \rho\right|^{2}\right)^{\frac{1}{2}}\right\|_{L_{t}^{q}\left(\mathbb{R} ; L_{r^{n-1} \mathrm{~d} r}^{q}\right.}([R, 2 R])\right) \\
& \lesssim\left\|r^{-\frac{n-2}{2}}\left(\sum_{k=0}^{\infty} \sum_{\ell=1}^{d(k)}\left\|\frac{1}{2 \pi} \int_{I_{2}} e^{2 \pi i \rho r \Phi_{2 \pi \rho r, k_{n}}(\theta)} \mathrm{d} \theta \sum_{j} b_{k, \ell}^{j} e^{i \frac{\pi}{2} \rho j} \varphi(\rho)\right\|_{L_{\rho}^{q^{\prime}}}^{2}\right)^{\frac{1}{2}}\right\|_{L_{r^{n-1} \mathrm{~d} r}^{q}([R, 2 R])} .
\end{aligned}
$$

Since supp $\varphi \subset\left[\frac{1}{2}, 4\right]$, it follows from (3.14) and Hölder's inequality that

$$
\begin{gathered}
\left\|r^{-\frac{n-2}{2}}\left(\sum_{k=0}^{\infty} \sum_{\ell=1}^{d(k)}\left|\int_{0}^{\infty} e^{-2 \pi i t \rho} \tilde{J}_{k_{n}}^{2}(2 \pi r \rho) \sum_{j} b_{k, \ell}^{j} e^{i \frac{\pi}{2} \rho j} \varphi(\rho) \mathrm{d} \rho\right|^{2}\right)^{\frac{1}{2}}\right\|_{L_{t}^{q}\left(\mathbb{R} ; L_{r^{n-1} \mathrm{~d} r}^{q}([R, 2 R])\right)} \\
\lesssim\left(\int_{R}^{2 R} r^{n(1-q / 2)-1} \mathrm{~d} r\right)^{\frac{1}{q}} \lesssim R^{-n\left(\frac{1}{2}-\frac{1}{q}\right)} .
\end{gathered}
$$

- Contribution of the case $\theta \in I_{3}$. In this case, since $\left|\Phi_{r, k}^{\prime \prime}(\theta)\right| \geq \sin \delta$, the Van der Corput Lemma 2.1 again yields that

$$
\left|\frac{1}{2 \pi} \int_{I_{3}} e^{i r \Phi_{r, k}(\theta)}\left(1-\chi_{\delta}(\theta)\right) \mathrm{d} \theta\right| \leq c_{\delta} r^{-1 / 2}
$$

uniformly in $k$. Similar to arguments with $q \geq 2$, it follows from (3.16) and Hölder's inequality that

$$
\begin{gathered}
\left.\left\|r^{-\frac{n-2}{2}}\left(\sum_{k=0}^{\infty} \sum_{\ell=1}^{d(k)}\left|\int_{0}^{\infty} e^{-2 \pi i t \rho} \tilde{J}_{k_{n}}^{3}(2 \pi r \rho) \sum_{j} b_{k, \ell}^{j} e^{i \frac{\pi}{2} \rho j} \varphi(\rho) \mathrm{d} \rho\right|^{2}\right)^{\frac{1}{2}}\right\|_{L_{t}^{q}\left(\mathbb{R} ; L_{r n-1}^{q} \mathrm{~d} r\right.}([R, 2 R])\right) \\
\lesssim\left(\int_{R}^{2 R} r^{(n-1)(1-q / 2)} \mathrm{d} r\right)^{\frac{1}{q}} \lesssim R^{-\frac{n-1}{2}\left(1-\frac{2 n}{q(n-1)}\right)}
\end{gathered}
$$


It is worth noting that we only use the oscillation given by $e^{2 \pi i t \rho}$ in terms of the Hausdorff-Young inequality. In the next case, we will use the oscillation given by $e^{2 \pi i t \rho}$ in a different argument.

- Contribution of the case $\theta \in 2 I_{1}$. To establish (3.10) in this case, we have to use effectively the oscillation of $e^{2 \pi i t \rho}$. For simplicity, we define

$$
\psi_{t-\frac{j}{4}}^{k}(r)=\frac{1}{2 \pi} \int_{0}^{\infty} e^{-2 \pi i\left(t-\frac{j}{4}\right) \rho} \int_{\mathbb{R}} e^{2 \pi i \rho r \sin \theta-i k_{n} \theta} \chi_{\delta}(\theta) \mathrm{d} \theta \varphi(\rho) \mathrm{d} \rho .
$$

Let $m=t-\frac{j}{4}$. Then we write

$$
\begin{aligned}
\psi_{m}^{k}(r) & =\frac{1}{2 \pi} \int_{\mathbb{R}^{2}} e^{2 \pi i \rho(r \sin \theta-m)} e^{-i k_{n} \theta} \chi_{\delta}(\theta) \varphi(\rho) \mathrm{d} \rho \mathrm{d} \theta \\
& =\frac{1}{2 \pi} \int_{\mathbb{R}} \check{\varphi}(r \sin \theta-m) e^{-i k_{n} \theta} \chi_{\delta}(\theta) \mathrm{d} \theta .
\end{aligned}
$$

For our purpose, we need to investigate the asymptotic behavior of the function $\psi_{m}^{k}(r)$. To this end, we consider the following two cases.

- Subcase $(a): 4 R \leq|m|$. Since $R \geq 1$, then $|m| \geq 4$. Since $\check{\varphi}$ is a Schwartz function, then

$$
|\check{\varphi}(r \sin \theta-m)| \leq C_{N}(1+|r \sin \theta-m|)^{-N}, \quad \forall N>0 .
$$

On the other hand, we have

$$
|r \sin \theta-m| \geq|m|-r|\sin \theta| \geq|m| / 100,
$$

by making use of $r \leq 2 R \leq|m|$ and $|\theta| \leq 2 \delta$. Thus, (3.19) gives that

$$
\left|\psi_{m}^{k}(r)\right| \leq C_{\delta, N}(1+|m|)^{-N}
$$

Hence keeping in mind that $m=t-\frac{j}{4}$, we have

$$
\begin{aligned}
& \left\|r^{-\frac{n-2}{2}}\left(\sum_{k=0}^{\infty} \sum_{\ell=1}^{d(k)}\left|\sum_{\left\{j: 4 R \leq\left|t-\frac{j}{4}\right|\right\}} b_{k, \ell}^{j} \psi_{t-\frac{j}{4}}^{k}(r)\right|^{2}\right)^{\frac{1}{2}}\right\|_{L_{t}^{q}\left(\mathbb{R} ; L_{r n-1 \mathrm{~d} r}^{q}([R, 2 R])\right)} \\
& \leq \frac{C_{\delta, N}}{R^{-N}}\left\|r^{-\frac{n-2}{2}}\left(\sum_{k=0}^{\infty} \sum_{\ell=1}^{d(k)}\left|\sum_{\left\{j: 4 R \leq\left|t-\frac{j}{4}\right|\right\}}\right| b_{k, \ell}^{j}\left|\left(11+\left|t-\frac{j}{4}\right|\right)^{-N}\right|^{2}\right)^{\frac{1}{2}}\right\|_{L_{t}^{q}\left(\mathbb{R} ; L_{r n-1}^{q}([R, 2 R])\right)} .
\end{aligned}
$$

By the Cauchy-Schwarz inequality and choosing $N$ large enough, the above is bounded by

$$
\begin{aligned}
& C_{\delta, N} R^{-N}\left\|r^{-\frac{n-2}{2}}\left(\sum_{k=0}^{\infty} \sum_{\ell=1}^{d(k)} \sum_{j}\left|b_{k, \ell}^{j}\right|^{2}\left(1+\left|t-\frac{j}{4}\right|\right)^{-N}\right)^{\frac{1}{2}}\right\|_{L_{t}^{q}\left(\mathbb{R} ; L_{r^{n-1} \mathrm{~d} r}^{q}([R, 2 R])\right)} \\
& \leq C_{\delta, N} R^{-N}\left(\sum_{k=0}^{\infty} \sum_{\ell=1}^{d(k)} \sum_{j}\left|b_{k, \ell}^{j}\right|^{2}\left(\int_{\mathbb{R}}\left(1+\left|t-\frac{j}{4}\right|\right)^{-\frac{q N}{2}} \mathrm{~d} t\right)^{2 / q}\right)^{\frac{1}{2}} \lesssim R^{-N} .
\end{aligned}
$$

- Subcase $(b):|m|<4 R$. Again since $\check{\varphi}$ is a Schwartz function, then

$$
|\check{\varphi}(r \sin \theta-m)| \leq C_{N}(1+|r \sin \theta-m|)^{-N} \quad \forall N>0 .
$$


By (3.19), this gives that

$$
\begin{aligned}
\left|\psi_{m}^{k}(r)\right| \leq & \frac{C_{N}}{2 \pi}\left(\int_{\{\theta:|\theta|<2 \delta,|r \sin \theta-m| \leq 1\}} \mathrm{d} \theta\right. \\
& \left.+\int_{\{\theta:|\theta|<2 \delta,|r \sin \theta-m| \geq 1\}}(1+|r \sin \theta-m|)^{-N} \mathrm{~d} \theta\right) .
\end{aligned}
$$

Let $y=r \sin \theta-m$. Then we have

$$
\left|\psi_{m}^{k}(r)\right| \leq \frac{C_{N}}{2 \pi r}\left(\int_{\{y:|y| \leq 1\}} \mathrm{d} y+\int_{\{y:|y| \geq 1\}}(1+|y|)^{-N} \mathrm{~d} y\right) \lesssim \frac{1}{r} .
$$

For fixed $t, R$, we define the set $A=\left\{j \in \mathbb{Z}:\left|t-\frac{j}{4}\right| \leq 4 R\right\}$. It is easy to see that the number of elements belonging to $A$ is $O(R)$. Thus it follows from (3.22) and the Cauchy-Schwarz inequality that

$$
\begin{array}{r}
\left\|r^{-\frac{n-2}{2}}\left(\sum_{k=0}^{\infty} \sum_{\ell=1}^{d(k)}\left|\sum_{j \in A} b_{k, \ell}^{j} \psi_{t-\frac{j}{4}}^{k}(r)\right|^{2}\right)^{\frac{1}{2}}\right\|_{L_{t}^{\infty}\left(\mathbb{R} ; L_{r^{n-1} \mathrm{~d} r}^{\infty}([R, 2 R])\right)} \\
\leq C_{\delta, N} R^{-\frac{n-1}{2}}\left(\sum_{k=0}^{\infty} \sum_{\ell=1}^{d(k)} \sum_{j}\left|b_{k, \ell}^{j}\right|^{2}\right)^{\frac{1}{2}} \lesssim R^{-\frac{n-1}{2}} .
\end{array}
$$

This together with (3.21) yields that (3.10); hence we complete the proof of Proposition 3.2

\section{ACKNOWLEDGMENTS}

The authors would like to express their gratitude to S. Shao for his helpful discussions and valuable suggestions. The authors also thank the referee and the associate editor for their invaluable comments and suggestions, which helped improve the paper greatly. The authors were partly supported by the NSF of China (No. 10725102).

\section{REFERENCES}

[1] B. Barcelo. On the restriction of the Fourier transform to a conical surface, Trans. Amer. Math. Soc. 292 (1985), 321-333. MR805965 (86k:42023)

[2] F. Nicola, Slicing surfaces and Fourier restriction conjecture, Proceedings of the Edinburgh Mathematical Society 52 (2009), 515-527. MR2506403

[3] S. Shao, Sharp linear and bilinear restriction estimates for paraboloids in the cylindrically symmetric case, Revista Matemática Iberoamericana 25 (2009), 1127-1168. MR2590695

[4] S. Shao, A note on the cone restriction conjecture in the cylindrically symmetric case, Proc. Amer. Math. Soc. 137 (2009), 135-143. MR2439434 (2010f:35222)

[5] E. M. Stein, Some problems in harmonic analysis, Harmonic analysis in Euclidean spaces (Proc. Sympos. Pure Math., Williams College, Williamstown, Mass., 1978), Amer. Math. Soc., 1979, pp. 3-20. MR.545235 (80m:42027)

[6] E. M. Stein, Harmonic Analysis: Real Variable Methods, Orthogonality and Oscillatory Integrals, Princeton Mathematical Series, 43, Princeton University Press, Princeton, N.J., 1993. MR1232192 (95c:42002)

[7] E. M. Stein and G. Weiss, Introduction to Fourier Analysis on Euclidean Spaces, Princeton Mathematical Series, 32, Princeton University Press, Princeton, N.J, 1971. MR.0304972 $(46: 4102)$

[8] R. Strichartz, Restrictions of Fourier transforms to quadratic surfaces and decay of solutions of wave equations. Duke. Math. J. 44 (1977), 705-714. MR0512086 (58:23577)

[9] J. Sterbenz, Appendix by I. Rodnianski, Angular regularity and Strichartz estimates for the wave equation. Int. Math. Res. Notices 4 (2005), 187-231. MR2128434 (2006i:35212) 
[10] T. Tao, Recent progress on the restriction conjecture. arxiv:math/0311181.

[11] T. Tao, The Bochner-Riesz conjecture implies the restriction conjecture, Duke Math. J. 96 (1999), 363-375. MR1666558 (2000a:42023)

[12] T. Tao, Endpoint bilinear restriction theorems for the cone and some sharp null form estimates, Math. Z. 238 (2001), 215-268. MR.1865417 (2003a:42010)

[13] T. Tao, A sharp bilinear restrictions estimate for paraboloids, Geom. Funct. Anal. 13 (2003), 1359-1384. MR2033842 (2004m:47111)

[14] T. Tao, A. Vargas and L. Vega, A bilinear approach to the restriction and Kakeya conjectures, J. Amer. Math. Soc. 11 (1998), 967-1000. MR1625056 (99f:42026)

[15] G. N. Watson, A Treatise on the Theory of Bessel Functions. Second Edition, Cambridge University Press, 1944. MR0010746 (6:64a)

[16] T. Wolff, A sharp bilinear cone restriction estimate, Ann. of Math. (2) 153 (2001), 661-698. MR:1836285 (2002j:42019)

Institute of Applied Physics and Computational Mathematics, P.O. Box 8009, Beijing, People's Republic of China 100088

E-mail address: miao_changxing@iapcm.ac.cn

The Graduate School of China Academy of Engineering Physics, P.O. Box 2101, Beijing, People's Republic of China 100088

Current address: Department of Mathematics, Beijing Institute of Technology, Beijing, People's Republic of China 100081 - and - Beijing Computational Science Research, Beijing, People's Republic of China 100084

E-mail address: zhangjunyong111@sohu.com

The Graduate School of China Academy of Engineering Physics, P.O. Box 2101, Beijing, People's Republic of China 100088

E-mail address: zhengjiqiang@gmail.com 